\title{
Los programas de transferencia de renta desde la perspectiva de derechos: El caso de Chile Solidario
}

\author{
Ana María Contreras Duarte* \\ María Angélica Rodríguez Llona**
}

\begin{abstract}
RESUMEN
El Estado Chileno, en las últimas décadas, ha orientado su acción en el área social de las políticas públicas hacia la generación de un Sistema de Protección social que, en términos declarativos, apunta a generar condiciones para el disfrute y ejercicio de los derechos de las personas, concebidas como titulares de un conjunto de prerrogativas y facultades que el Estado debe respetar, proteger y promover.

Dicho sistema pareciera ser ampliamente incluyente tanto respecto de la población que abarca, como de los derechos y recursos que provee. No obstante, al observar algunas de las iniciativas que comprende, se aprecia que éstas darían cuenta de una opción por los sectores más pobres de la población, debiendo los otros grupos sociales satisfacer sus necesidades, cada vez más, a través del mercado privado de recursos y servicios. Además de los problemas de cohesión social que esta situación genera, el análisis documental de programas sociales orientados a grupos que viven en situaciones de extrema pobreza, permite interrogarse respecto de la capacidad que poseen los programas de transferencia de renta -en tanto componente fundamental del actual sistema de protección social chileno-, para abordar la superación de la pobreza extrema en el país. Para ello, en este artículo, se adopta la perspectiva de los Derechos Humanos en el análisis de los programas de transferencia de renta, examinando el cumplimiento de ciertos criterios que, desde este enfoque, permitan establecer la existencia concreta de una propuesta normativa y operativamente fundada para la promoción, protección y garantía de los derechos civiles, políticos, económico-sociales y culturales (DESC).
\end{abstract}

Palabras clave: Protección social - pobreza - políticas públicas - programas de transferencia de renta - ciudadanía - perspectiva de derechos.

1 Este artículo formó parte de una ponencia presentada por las autoras en el II Foro Latinoamericano de Trabajo Social "Escenarios de la vida social, el Trabajo Social y las Ciencias Sociales en el siglo XXI", realizado en La Plata, provincia de Buenos Aires, Argentina, entre los días 28 y 30 de agosto del 2008. En éste, se da cuenta de parte del debate teórico realizado a propósito de la formulación de un proyecto de investigación denominado: "Los programas de transferencia de renta como estrategia para enfrentar la pobreza en América Latina", presentado a concurso interno de investigación en la Universidad Católica Silva Henríquez.

* Chilena. Docente Departamento de Trabajo Social, Universidad Católica Silva Henríquez, Santiago de Chile. Correo electrónico: acontrer@ucsh.cl

** Chilena. Docente Departamento de Trabajo Social, Universidad Católica Silva Henríquez, Santiago de Chile. Correo electrónico: mrodrig@ucsh.cl 


\title{
Os programas de transferência de renda desde a perspectiva de direitos: O caso de Chile solidário
}

\begin{abstract}
RESUMO
O Estado Chileno, nas últimas décadas, orientou sua acção no área social das políticas públicas para a geração de um Sistema de Protecção social que, em termos declarativos, aponta a gerar condições para o desfrute e exercício dos direitos das pessoas, concebidas como titulares de um conjunto de prerrogativas e faculdades que o Estado deve respeitar, proteger e promover.

Dito sistema parecesse ser amplamente incluyente tanto respecto da população que abarca, como dos direitos e recursos que provee. Não obstante, ao observar algumas das iniciativas que compreende, se aprecia que estas dariam conta de uma opção pelos sectores mais pobres da população, devendo os outros grupos sociais satisfacer mercado privado de recursos e serviços. Além dos problemas de coesão social que esta situação gera, a análise documental de programas sociais orientados a grupos que vivem em situações de extrema pobreza, permite se interrogar respecto da capacidade que possuem os programas de transferência de renda -em tanto componente fundame examinando o cumprimento de certos critérios que, desde este enfoque, permitam estabelecer a existência concreta de uma proposta normativa e operativamente fundada para a promoção, protecção e garantia dos direitos civis, políticos, económicosociais e culturais (DESC).
\end{abstract}

Palavras chave: Protecção social - pobreza - políticas públicas - programas de transferência de renda - cidadania - perspectiva de direitos

\section{The income transfer programs from the perspective of rights: The case of Chile Solidario}

\begin{abstract}
The Chilean State, in recent decades has oriented its action in the social area of public policies towards the generation of a social protection system in declarative terms, aims to create conditions for the enjoyment and exercise of the rights of people, conceived as holding a set of prerogatives and powers that the State must respect, protect and promote.

This system appears to be broadly inclusive both in terms of population covered, as the rights and remedies provided. However, noting a number of initiatives including, we see that they would notice an option for the poorest segments of the population, other social groups must meet their needs, increasingly, through the private market resources and services. In addition to problems of social cohesion that this situation is creating the documentary analysis of social programs targeted to groups living in extreme poverty, some questions about the ability to have the income transfer programs-as fundamental component of the current Chilean social protection system, to address the eradication of extreme poverty in the country. In this article, we adopt the perspective of Human Rights in the analysis of income transfer programs, examining compliance with certain criteria, since this approach would establish the actual existence of a policy proposal and operationally based for the promotion, protection and guarantee of civil, political, economic, social and cultural rights (DESC).
\end{abstract}

Key words: Social welfare - poverty - public policies - income transfer programs - citizenship - the rights perspective 


\section{Antecedentes}

Las diversas transformaciones sociales observadas en el contexto de la denominada sociedad global, particularmente en la esfera de la organización social y política de los Estados, plantean nuevas preguntas en torno a las posibilidades del desarrollo en América Latina y el impacto que tienen las políticas sociales y de alivio a la pobreza. En este escenario, el tema de la protección social ha cobrado un creciente interés, generando procesos de reflexión que han permitido resignificar antiguos enfoques y levantar nuevas propuestas para la superación o mitigación de las consecuencias ocasionadas por el nuevo orden neoliberal.

Es posible diferenciar al menos tres momentos en la trayectoria de la política social en el continente durante el siglo XX, momento en que la preocupación por la cuestión social pasa de un enfoque privado y de beneficencia a un enfoque de política pública. Un primer momento, denominado enfoque tradicional, estaría marcado por la incursión del Estado en esta materia, hecho que coincide con lo que algunos han denominado "el desarrollismo latinoamericano", basado en un modelo de industrialización sustitutiva de importaciones. Según Franco (1996), este periodo persiguió la protección del trabajador asalariado, respondiendo a las presiones derivadas de la creciente organización sindical por la conquista de nuevos servicios sociales y mejoras en sus condiciones de vida. Así, el Estado impulsa políticas sectoriales que buscan ampliar el acceso de la población a una variada gama de servicios de educación, salud, previsión social, vivienda y urbanización, teniendo un carácter universalista. Un segundo momento se materializa en los años 80, momento marcado por la crisis fiscal y la instauración de regímenes autoritarios. La represión cambia por la fuerza el contrato social implícito y el paquete de políticas públicas que sustentaba la vieja alianza social entre los sectores medios y sindicales (Cortés, 1999). Este periodo, también denominado de ajuste estructural, conduce a una ofensiva neoliberal, que se difunde desde los organismos multilaterales, y que atribuye un rol protagónico al sector privado y al mercado, siendo sus ejes programáticos la privatización y la apertura al mercado exterior. En este periodo se observa una subordinación del gasto público -vía gasto social- a la política monetaria. La política social transita desde una concepción universalista, centrada en la oferta de los servicios sociales, a una de carácter focalizado, centrada en el 
subsidio a la demanda. Finalmente, el tercer momento, corresponde a los años 90, período en el que, en un gran número de países latinoamericanos, se restauran los gobiernos democráticos luego de dictaduras militares y se discuten nuevos enfoques y alternativas para asegurar un mayor equilibrio entre Estado, mercado y sociedad. Se privilegian programas destinados a la superación de la pobreza, dándole un mayor énfasis al crecimiento con equidad, pero sin transformar sustantivamente el modelo económico.

En este contexto, el Estado Chileno, a partir del año 2002, orienta su acción en el área social de las políticas públicas hacia la generación de un Sistema de Protección Social que aspira a generar condiciones para el disfrute de los derechos de las personas y protección para su efectivo ejercicio. El Sistema de protección social ha sido entendido, desde el nivel estatal, como "el mecanismo que permite generar condiciones que brinden seguridad a las personas durante toda su vida, garantizándoles derechos sociales que les permitan finalmente reducir los riesgos en empleo, salud, educación y previsión, generando condiciones de mayor igualdad y oportunidades de progreso" (MIDEPLAN, 2008). Así definido, el sistema pareciera ser ampliamente incluyente, no solo respecto de la población que abarca, sino de los derechos y recursos que provee. Sin embargo, las iniciativas que éste comprende dan cuenta de una opción por los grupos más pobres de la población, debiendo los otros grupos sociales satisfacer, cada vez más, sus necesidades a través del mercado privado de recursos y servicios. En este sentido, De la Maza, en el 2004, ya advierte del abandono que ha ido haciendo el Estado chileno de los grupos socio-económicos medios al optar por la focalización como estrategia fundamental del gasto social. Para el autor, el Estado favorece el vínculo con aquel segmento de la población más desfavorecida, a través de la política social de vivienda, previsión social, salud y educación: "La creciente focalización de dichas políticas en pobreza las ha hecho abandonar la atención de grupos medios, quienes son atendidos crecientemente por mecanismos de mercado" (De la Maza, 2004:12). Se observa cada vez más la renuncia hacia la universalización de un sistema de protección que garantice los derechos sociales económicos, sociales y culturales para todos los ciudadanos, debiendo las personas asumir, de manera individual y privada, la resolución de las necesidades fundamentales y el acceso a diversos servicios que en el pasado fueron atribución del Estado. 
Lo anterior precariza cada vez más la situación de protección de derechos sociales, económicos y culturales para los ciudadanos en Chile. Estar protegido, para Robert Castel (2004), supone que las personas dispongan, desde su nacimiento y por derecho propio, de las condiciones sociales y materiales mínimas para su desarrollo. Es la condición ineludible para una sociedad de semejantes, equitativa, donde todo integrante dispone de un piso de derechos que asegura su inclusión desde el inicio de la vida. ${ }^{2}$ Para el autor, la seguridad social mínima garantizada es necesaria como condición de la ciudadanía social, y por ello, debería incluir e involucrar a todo el mundo, ya que todos la necesitan y no sólo los más pobres, porque justamente sin esos derechos sociales de base, los individuos son dejados a sí mismos y quedan completamente sin recursos (Castel, 2005). Por tanto, una de las características fundamentales de un sistema de protección debiera ser su capacidad inclusiva, otorgando a todas las personas posibilidades para la realización de sus derechos fundamentales y cobertura a un conjunto de necesidades sociales y económicas vitales para alcanzar niveles dignos de desarrollo, dado que el mercado ha resultado insuficiente como mecanismo de realización de derechos, especialmente en países en los que se observa una gran desigualdad económica, como es el caso chileno. ${ }^{3}$

Respecto de las prestaciones o recursos de que debe proveer un sistema de protección social, los autores distinguen al menos dos aspectos o componentes: el aseguramiento y la asistencia social (Guerrero, 2006). En las sociedades modernas, el aseguramiento ha estado ligado al contrato de trabajo, por lo que un desafío de la política pública es que todos los ciudadanos, posean o no la categoría de trabajadores, tengan acceso a instrumentos adecuados de aseguramiento, lo que es fundamental en contextos de incertidumbre como los actuales. El componente asistencial del sistema de protección, por otra parte, busca mitigar las consecuencias producidas por situaciones de pobreza y vulnerabilidad que afectan a la población. El Estado, a través de diversos programas sociales, otorga recursos materiales y subsidios monetarios para el desarrollo de este componente del sistema de seguridad social.

2 Citado por: Sistemas de protección social de infancia y adolescencia: desafíos y posibilidades. Instituto del niño y el adolescente de Uruguay, INAU, 2007.

3 En Chile, la brecha entre el diez por ciento más rico y el diez por ciento más pobre es del orden de 43 veces o, según el Banco Mundial y el PNUD, de 25-36 veces (Kremerman, Marcos. Fundación Terram, 2005). 


\section{La perspectiva de derechos como marco moral y normativo de la formulación de las políticas sociales orientadas a la superación de la pobreza}

Los programas de transferencia de renta (PTR), como estrategia para enfrentar la pobreza y en tanto componentes del sistema de protección social, se convierten en un campo interesante de discusión en términos de su eficacia para lograr la integración social y su capacidad para garantizar derechos de los ciudadanos/ as. Las preocupaciones vienen dadas por el carácter universal que se debiera otorgar a los derechos sociales, económicos y culturales (DESC) y la efectiva progresividad en el logro de satisfactores para su concreción, establecida como característica de esta generación de derechos. Este último rasgo permite a cada Estado la realización de los DESC de acuerdo a sus recursos y posibilidades para la definición de contenidos mínimos efectivamente exigibles para todos los ciudadanos/as.

Para dilucidar la manera en que las opciones políticas y económicas del Estado chileno favorecen el goce de estos derechos y otorgan garantías mínimas para su exigibilidad, se adopta en este artículo lo que se ha venido llamando el enfoque de derechos humanos. Este enfoque adquiere cada vez más importancia, en la medida que no sólo constituye un referente moral que dota de un sentido declarativo a las políticas, sino también en un marco normativo para la formulación de las mismas. Tal como lo señala la Oficina del Alto Comisionado de las Naciones Unidas para los Derechos Humanos -OACNUDH-, el enfoque de derechos humanos ofrece un marco normativo explícito, a saber, el de los derechos humanos en el ámbito internacional: “... respaldados por valores morales universalmente reconocidos y reforzados por las obligaciones jurídicas, los derechos humanos constituyen en el plano internacional un marco normativo imprescindible para la formulación de políticas nacionales e internacionales, incluidas las estrategias de reducción de la pobreza" (2006). 
Para la CEPAL, en una perspectiva de derechos ${ }^{4}$ y ciudadanía, es deber del Estado la garantía amplia de todos los derechos y no sólo los civiles y políticos, que corresponden a la primera generación de derechos: los Estados deben asumir responsabilidades en la garantía de derechos de todos los ciudadanos de la república. Respecto de su contenido, comprenderían, además de los civiles y políticos, los derechos sociales que constituyen la ciudadanía social (CEPAL, 2000). El Estado, entonces, debe ser capaz de crear condiciones que permitan no sólo el goce de los derechos civiles y políticos, absteniéndose de perturbar o amenazar estos derechos o su ejercicio, sino también debe procurar un conjunto de acciones y mecanismos que permitan el goce de los derechos económicos, sociales y culturales -o de segunda generación- y su exigibilidad frente al Estado y otros sectores de la sociedad involucrados en su desarrollo y concreción.

Se parte de la base que los derechos económicos, sociales y culturales están plenamente reconocidos por la legislación internacional de derechos humanos, por lo tanto, el foco de discusión no es su estatuto de derechos humanos básicos, sino a qué dan derecho y al carácter jurídico que tienen las obligaciones de los Estados. El enfoque basado en la universalidad de los derechos humanos fundamentales propone el acceso universal a los servicios públicos, siendo la ciudadanía el concepto que articula el acceso y ejercicio de estos derechos. En este sentido, Delamónica (2000) sostiene que el acceso a los servicios es necesario para el ejercicio efectivo de los mismos y la construcción de ciudadanía en sociedades incluyentes.

La incorporación de esta perspectiva en el diseño de políticas permite fundarlas, no solo desde las necesidades, sino desde los derechos que tienen quienes viven en situación de pobreza, creando obligaciones jurídicas para terceros. "De esta manera, la perspectiva de los derechos humanos pone de relieve que la pobreza trae consigo la no realización de los derechos humanos, con lo cual la adopción de una estrategia de reducción de la pobreza es no sólo deseable

4 En el marco de las discusiones contemporáneas sobre desarrollo, ganó terreno en la década de 1990 la idea de corregir las limitaciones y errores de las propuestas de modernización productiva, introduciendo una consideración fuerte sobre los derechos. Antecedentes se encuentran en Human Rights Council of Australia, 1995 y 1998. También en la adopción, en 1986, de la Declaración en torno al Derecho al Desarrollo, promovida por los países en vías de desarrollo, que establecía un vínculo fuerte entre Desarrollo y Derechos. 
sino también obligatoria para los Estados que han ratificado los instrumentos internacionales de derechos humanos." (ACNUDH, 2006).

El enfoque de derechos humanos hace hincapié en la importancia de esos derechos en todas las esferas sociales y normativas, incorporándolos como requerimientos básicos en el proceso de formulación de políticas orientadas a la reducción de la pobreza y a alcanzar mejores niveles de desarrollo. Según la Oficina del ACNUDH, se trata de "un marco conceptual para el proceso de desarrollo humano que está normativamente fundado en los estándares internacionales de derechos humanos y operativamente dirigido a promover y proteger estos derechos" (ACNUDH, 2006). El análisis de este marco conceptual permite relevar las principales características del enfoque de derechos humanos: su orientación hacia el empoderamiento de las personas, ampliando su libertad de opción y acción para estructurar sus vidas, facultando a los individuos y comunidades al otorgarles derechos que imponen obligaciones a otros, entre éstos, a los Estados. Además, la adopción de este enfoque implica el reconocimiento explícito de un marco normativo de derechos humanos a nivel nacional e internacional, la ampliación de las responsabilidades judiciales, administrativas y políticas de los distintos actores involucrados en los procesos de desarrollo, de modo que los derechos no se conviertan en meras declaraciones, sino en garantías de un trato no discriminatorio e igualitario. Sumado a lo anterior, un enfoque de derechos humanos debe caracterizarse por la participación activa e informada de las personas en las distintas fases del proceso de generación e implementación de las diversas políticas públicas orientadas a la superación de la pobreza y al logro del desarrollo. Respecto de la progresividad, como característica del enfoque, es necesario señalar que ésta permite un horizonte temporal para evaluar la progresión y el establecimiento de ciertas prioridades, sin perjuicio de la definición de ciertos niveles esenciales mínimos exigibles de inmediato (ACNUDH, 2004).

De ahí que, en lo que respecta al carácter de las prestaciones a que dan origen las políticas sociales, no se trata de la entrega asistencial de algunos recursos, sino que la realización de las necesidades humanas se constituya en un derecho a contar con los mecanismos sociales necesarios para facilitar el acceso a su satisfacción. Se 
reconoce que la plena realización de todos los derechos sociales, culturales y económicos (DESC) no es un objetivo que se pueda cumplir en forma inmediata, especialmente en países con un menor nivel de desarrollo y bajo ingreso. Lo que se requiere es una realización gradual mediante el progreso social y económico de largo plazo. Por tanto, la obligación del Estado es hacer todo lo que esté a su alcance por promover el progreso lo más rápidamente posible. Su responsabilidad se juzgará no sólo por la realización o no de un derecho, sino también por si se han formulado y aplicado políticas efectivas y si se están haciendo progresos en esta materia.

\section{El caso de Chile Solidario}

El gobierno de Chile, desde inicios de la década del 2000, recoge el concepto de protección social desarrollado por el Banco Mundial, a través del Chile Solidario, principal programa de gobierno dirigido a la extrema pobreza en el país. El objetivo que se planteó el programa, en sus inicios, es "mejorar las condiciones de vida de 225 mil familias en extrema pobreza, generando las oportunidades y proveyendo los recursos que permitan recuperar o disponer de una capacidad funcional y resolutiva eficaz en el entorno personal, familiar, comunitario e institucional" (MIDEPLAN, 2002). Este sistema se orienta a entregar, de manera organizada y coordinada, la oferta programático-institucional, asistencial y promocional que ofrece el Estado, otorgando prestaciones asistenciales garantizadas a las familias. Se estructura en torno a 53 prestaciones sociales organizadas en siete ámbitos de derecho: salud, educación, vivienda, trabajo, ciudadanía, cultura y derecho a una vida familiar plena. Para ello, el sistema actúa en tres etapas: la primera de ellas es el "Acompañamiento", cuya principal característica estaría dada por el apoyo psicosocial que presta un operador social. En esta etapa, las familias, a través de la firma de un contrato, se comprometen a trabajar con un profesional denominado "apoyo familiar" durante 24 meses, para el cumplimiento de ciertos requisitos mínimos en cada uno de los ámbitos de derecho antes mencionados. Esta etapa es apoyada con un bono monetario, asociado al cumplimiento del contrato, que va decreciendo en el tiempo y que comienza con \$ 12.320 (US\$ 22,7) y finaliza con \$ 5.765 (US\$ 10.6) ${ }^{5}$. La segunda

5 Valores actualizados a agosto del 2008. Fecha de cálculo de su equivalencia en dólares: 30/07/09. 
etapa corresponde a lo que se ha denominado "Inserción Social", consistente en la incorporación de la familia a la red social existente, particularmente a través de un acceso preferente a ciertos subsidios monetarios garantizados ${ }^{6}$. Finalmente, se encuentra la etapa de "Desarrollo", que apunta a generar las condiciones básicas de habilitación de las familias para iniciar el fortalecimiento del capital social, mediante un acceso preferente a programas de promoción social.

No obstante las evaluaciones positivas realizadas al Programa Chile Solidario, en el sentido que ha permitido rearticular la oferta pública, mejorar la coordinación institucional y el impacto de los programas sociales y las políticas sectoriales, persisten en él una serie de falencias que se aprecian, sobre todo, al analizarlo desde una perspectiva de derechos.

En primer lugar, con relación al marco normativo, y considerando el sustento legal de estos derechos, es necesario precisar en qué medida éstos constituyen una concesión por parte del Estado o más bien derechos efectivamente garantizados y exigibles. En el caso del Programa analizado, se observa un sustento legal básico, ya que además de la normativa presupuestaria que asegura su financiamiento, se promulga la ley 19949, que crea el Chile Solidario, todo lo cual es posible en virtud de un consenso político mínimo que buscó comprometer a los diversos actores de la política pública no sólo como prestadores, sino como garantes efectivos de la realización del contenido de los derechos establecidos en dicha ley. Así, el sistema de protección social Chile Solidario reconoce y asegura a las familias que se encuentran en condición de extrema pobreza una base esencial de derechos, planteando, como deber del Estado, la efectiva accesibilidad y eficiencia en la transferencia de recursos y prestaciones que impliquen el logro y realización de los DESC para la población señalada. De esta manera, los programas sociales, que en una etapa inicial fueron implementados por vía administrativa, han pasado a una segunda etapa, de carácter legislativo, en la que se consolidan como derechos algunas de las medidas contenidas en éstos. En este sentido, existe un cambio positivo en el camino hacia una comprensión más amplia de las responsabilidades progresivas

6 Subsidio Único Familiar (SUF), Subsidio a la Cédula de Identidad, Subsidio al Pago del Consumo de Agua Potable y Servicio de Alcantarillado de Aguas Servidas (SAP), Subvención Preferencial y Subvención Pro Retención Escolar y Pensión Básica Solidaria. 
que le caben al Estado en la promoción y protección de los derechos humanos. Sin embargo, está pendiente, como bien señala RuizTagle (2005), una tercera etapa en la que estos derechos emanados de los programas sociales puedan ser reconocidos y garantizados por los tribunales chilenos y hechos valer ante organismos públicos o privados.

Respecto de la progresividad y realización de los derechos, el Programa Chile Solidario, como ya se señaló, establece 53 mínimos sociales de distinto alcance, que si bien permiten una cobertura de protección e integración básica para las personas en situación de indigencia, evidencian la complejidad de su sostenibilidad en el tiempo, en la medida que los derechos o beneficios que cubre no afectan radicalmente la estructura del empleo y no aseguran una inserción laboral estable y con ingresos dignos a sus destinatarios ${ }^{7}$. Además, los montos de las rentas condicionadas que considera el programa son muy bajos, lo que refuerza su carácter asistencial y no modifica la situación de vulnerabilidad social de estos grupos sociales. Por otra parte, no se observa un incremento ascendente en términos de mayor accesibilidad a nuevas prestaciones que impliquen una mejor realización de estos derechos en el tiempo, más allá de los mínimos garantizados.

Los mínimos establecidos en el programa, al mismo tiempo, pueden ser también analizados desde el impacto que esta restrictiva definición de derechos ciudadanos tiene en la sociedad, en la medida que se naturaliza lo mínimo como lo ético y socialmente aceptable, normalizando estos estándares reducidos como condiciones y situaciones suficientes para la integración social. Como bien ha señalado Álvarez (2005), este discurso "minimista" remite a una nueva utopía que promueve una sociedad que, junto con lamentar la pobreza, no pone en cuestión los mecanismos básicos que la producen, limitando las políticas sociales a un umbral de ciudadanía que se reduce a parámetros de sobrevivencia. En otros términos, la política social de abordaje de la extrema pobreza, expresada en los

7 Para mayores antecedentes sobre estos aspectos del Chile Solidario, ver: Ruz, Miguel Ángel y Palma, Julieta "Análisis del proceso de elaboración e implementación del sistema del Chile Solidario", Instituto de Asuntos Públicos, Departamento de Políticas Públicas, Universidad de Chile, 2005; y Serrano, Claudia y Raczynski, Dagmar, "Derechos sociales básicos, superación de la pobreza y protección social ante la vulnerabilidad", Asesorías para el Desarrollo, Santiago de Chile, 2003. 
programas de transferencia de renta, no modifica la distribución de la riqueza al interior de los países, aspecto que, junto al fortalecimiento de la participación social y el reconocimiento de la especificidad social y cultural de las personas, constituye uno de los tres vértices fundamentales de la cohesión social, vista desde una perspectiva de derechos (CEPAL, 2007).

Un segundo aspecto, en términos de realización de derechos, refiere a la tensión entre focalización y universalidad de las políticas sociales. El Chile Solidario constituye un programa dirigido a la extrema pobreza en Chile, es decir, alrededor de un 3\% de la población chilena. En este contexto, es necesario determinar en qué medida el establecimiento de políticas sociales focalizadas y compensatorias se contraponen al carácter universal de los DESC, limitando las posibilidades y el disfrute de derechos que éstos contienen. Los postulados del enfoque del manejo del riesgo social, que a nuestro juicio está en la base de los programas de transferencia de renta, delimitan, según Sojo (2006), una responsabilidad pública mínima en materia de protección social por dos vías: la de circunscribir las responsabilidades del Estado sólo al combate de la pobreza, mediante redes de protección y contraponer las políticas focalizadas a las universales; y en el plano del aseguramiento, la de desestimar y socavar la solidaridad y hacer hincapié en la responsabilidad individual para asegurarse contra los riesgos.

En este último sentido, es posible constatar en estas propuestas de intervención, además de un reduccionismo de la acción y responsabilidad estatal, una incidencia que impacta no solo al grupo social en el cual se focaliza su acción, sino también a otros que viven en similares condiciones, pero que no califican para el programa. Ello incide negativamente en las relaciones entre grupos de un mismo sector, debilitando aún más las posibilidades de cohesión social de la sociedad chilena. En efecto, la focalización al interior de los grupos en situación de pobreza ha producido fragmentación social, por el agravio comparativo que significa que sólo algunos pobres tengan acceso a los beneficios de la red social, "dañando la confianza mutua y la formación de capital social precisamente en países donde ambos son más necesitados" (Moreno, 2007: 13).

En lo que respecta a la dimensión de participación social de los sujetos en el diseño e implementación de políticas sociales, es 
posible señalar que el programa Chile Solidario tiene una visión restringida de la participación social. En términos operativos, ésta se expresa fundamentalmente en la firma de un contrato familiar, sujeto al cumplimiento por parte de las familias de ciertas tareas orientadas al logro de mínimos establecidos en el Chile Solidario. Ello hace que la participación de las familias quede restringida a un contrato de adhesión al programa y no la definición de contenidos, criterios y requerimientos que se adecuen a la realidad y necesidades de las mismas y que recojan sus propias visiones y propuestas en torno a los procesos a desarrollar para salir de la pobreza. Del mismo modo, el programa tampoco moviliza sustancialmente los aspectos políticos de la pobreza, en el sentido que la dimensión promocional del Chile Solidario se ve sobrepasada con el componente asistencial del mismo, generándose situaciones de dependencia y pasividad entre los usuarios. Al mismo tiempo, éstos corren el alto riesgo de transformarse en mecanismos de clientelismo político, práctica bastante corriente en América Latina, particularmente en épocas electorales. A pesar de que existen instrumentos objetivos que apuntan a medir objetivamente las necesidades de la población en situación de pobreza (la ficha de protección social en el caso chileno), algunos estudios muestran que pese a la creación de estos catastros, hay evidencias de quejas por el uso discrecional que se hace de los recursos, los que no siempre llegan a quien más los necesita (Ruiz, 2008:128).

De este modo, la acción hacia el empoderamiento de los sujetos, que constituye uno de los requerimientos del enfoque basado en la perspectiva de derechos humanos, se observa debilitada, en la medida que se reducen las posibilidades de participación concreta de los sujetos en la toma de decisiones, en el diseño e implementación del programa, así como también en la ausencia de cuestionamiento sobre los factores más estructurales que originan la pobreza en Chile. Así, la integración de los beneficiarios es precaria y no modifica sustancialmente las posibilidades de éstos de ampliar su libertad de opción y acción para estructurar sus vidas.

Por otro lado, se construyen discursos sobre la realidad social que alcanzan la vida cotidiana de los sujetos con los cuales se interviene, denominándolos como los "más pobres entre los pobres", "no habilitados", "vulnerables", "las familias puente”, etcétera (Ovando 2007: s/p), institucionalizándose de este modo la violencia simbólica 
desde el Estado y las estrategias de intervención social que promueve para el enfrentamiento de las situaciones de pobreza.

\section{Conclusiones}

Hasta aquí se ha podido observar entonces que el programa Chile Solidario debe sortear aún importantes desafíos para cumplir con los principios orientadores de la perspectiva de derechos humanos, toda vez que esta constituye la opción formalmente declarada en su descripción oficial: "Este programa constituye una poderosa señal del enfoque de derechos garantizados que el presente Gobierno le ha dado a sus políticas sociales, buscando transitar desde una perspectiva de satisfacción de necesidades hacia otra de preservación de derechos sociales" (MIDEPLAN 2007: S7p) ${ }^{8}$.

El trabajo realizado por el gobierno de la actual presidenta Michèle Bachellet, constituye un progreso en materia de extensión y universalidad de derechos sociales. Las reformas al sistema de previsión social, el establecimiento del sistema AUGE -que incorpora garantías explícitas, es decir, derechos exigibles por las personas en la atención de 56 enfermedades o problemas de salud considerados prioritarios-, constituyen avances significativos en materia de protección social. Los programas de abordaje a la pobreza también forman parte de ampliación de derechos. No obstante, en el caso del Programa Chile Solidario, aún se requiere enfrentar una serie de desafíos.

En primer lugar, nos enfrentamos a una construcción de diversos discursos enfocados desde los derechos; sin embargo, se observa la presencia de fuertes condicionamientos y control social al momento de hacerlos efectivos, mediante el acceso a los recursos ofertados por el Estado en la modalidad de políticas y programas sociales. Ello exige, por ende, mejorar los mecanismos de exigibilidad de los derechos, generando jurisprudencia que fundamente las decisiones de los tribunales en la perspectiva de los derechos humanos.

En segundo término, el programa debe proponerse incrementar, de manera significativa, los contenidos de los beneficios establecidos en el mismo, de modo que, efectivamente, se cumpla con el principio

8 MIDEPLAN, 2007. Tomado de:

http://www.mideplan.cl/final/categoria. php?secid=1 \& catid=1 12 (Consultadoel3/10/2008). 
de progresividad en la satisfacción de los derechos económicos, sociales y culturales a los que chile se ha comprometido internacionalmente. Ello permitirá modificar de manera más real la situación de vulnerabilidad social de los sectores en situación de pobreza y ampliar la mirada restrictiva y minimalista que todavía existe en términos de definición de derechos ciudadanos, en el sentido del establecimiento de umbrales que restringen la ciudadanía de los grupos pobres a parámetros de sobrevivencia. Lo anterior supone un debate social serio respecto de los mecanismos básicos que producen la pobreza y la voluntad política para modificar la distribución de la riqueza al interior de los países, abordando particular e integralmente la problemática del empleo.

El programa, como se sostuvo, estimula situaciones de dependencia y pasividad entre los usuarios, por el de hecho que la dimensión promocional que lo inspira se ve completamente sobrepasada por su dimensión asistencial. Al mismo tiempo, fragmenta a los propios sectores pobres, debilitando los lazos comunitarios y las posibilidades de cohesión social. En ese sentido, es imperativo recrear e idear incentivos para fortalecer el capital social, la participación social y la asociatividad de las familias, reduciendo la resolución individual de los problemas que podrían ser enfrentados con mayor eficacia desde una perspectiva colectiva y solidaria.

En cuarto lugar, son cuestionables también las posibilidades de empoderamiento de los sujetos destinatarios del Chile Solidario, en la medida que el sistema sólo considera la información básica de servicios como estrategia de participación, sin desarrollar competencias efectivas para la toma de decisiones vitales y sin generar oportunidades concretas de influencia en el tipo de programa, relaciones y servicios que se establecen. En ese sentido, el programa enfrenta el desafío de establecer mecanismos eficaces que superen la mera adhesión de los sujetos al mismo y que impliquen una real actoria social de éstos, garantizándoles verdaderas posibilidades de inclusión social.

Finalmente, si bien el programa ha logrado una importante articulación de la red de protección social en beneficio de los destinatarios del programa, a través de un soporte tecnológico y computacional moderno y eficiente que permite el seguimiento de las acciones, todavía se requiere fortalecer y mejorar la calidad de la 
atención de las familias por parte de los profesionales y técnicos, así como de los servicios que componen la red social, de manera que no se requiera de la constante mediación del operador social del Chile Solidario (apoyo familiar) u otros profesionales para acceder a las prestaciones vinculadas a los derechos de que son titulares las personas destinatarias de este programa social

\section{Referencias bibliográficas}

Álvarez Leguizamón, Sonia (compiladora) (2005). “Trabajo y producción de la pobreza en Latinoamérica y el Caribe: estructuras, discursos y actores". Consejo Latinoamericano de Ciencias Sociales CLACSO. Disponible desde Internet: http:// bibliotecavirtual.clacso.org.ar/ar/libros/crop/ Trabprod.pdf

Castel, Robert (2005). Reflexiones sobre la relación: Estado, mercado, neo-liberalismo, trabajador móvil y seguridad social. Entrevista realizada por Pablo ChiesaNadia Schiavinato. Tomado de http://www. enredando.org.ar/noticias_desarrollo. shtml?x=26181

Comisión económica para América Latina, CEPAL (2000). Equidad, desarrollo y ciudadanía. Santiago de Chile.

Comisión económica para América Latina, CEPAL (2007). Cohesión social. Inclusión y sentido de pertenencia en América Latina y El Caribe. Santiago de Chile.

Cortés, Patricia (1999). La política social en América Latina. Notas para su evaluación al final del milenio. Revista Perspectivas $\mathrm{N}^{\circ}$ 8, Departamento de Trabajo Social, Universidad Católica Silva Henríquez, Santiago de Chile.

De la maza, Gonzalo (2004). Políticas Públicas y Sociedad Civil en Chile. El caso de las políticas Sociales (1990- 2004). En: Política $N^{\circ} 43$, Primavera 2004. INAP - U. de Chile, Santiago de Chile.

Delamónica (2000), en Sottoli, Susana (2002). La política social en América Latina. Diez dimensiones para el análisis y el diseño de políticas. Revista Papeles de población. UNAM. México.

Franco, Rolando (1996). Los paradigmas de la política social en América 
Latina, Revista CEPAL $N^{\circ}$ 58, Santiago de Chile.

Gobierno de Chile, Ministerio de Planificación y Cooperación, MIDEPLAN (enero, 2002). Estrategia de intervención integral a favor de familias en extrema pobreza, Santiago de Chile.

Gobierno de Chile, Ministerio de Planificación y Cooperación, MIDEPLAN (2008). Disponible desde Internet:

http://www.mideplan.cl/final/categoria. php?catid=1 10Esecid=39

Guerrero, Ramiro (2006) El concepto de protección social. Cuadernos de protección social

No 3 ,Bogotá Colombia.

INAU, Instituto del niño y el adolescente de Uruguay (2007). Disponible desde Internet: http://www.iberchile.cl/ prontus_iberchile/site/artic/20070416/ asocfile/20070416140701/sistemas_ de_proteccion_social_de_infancia_y_ adolescencia_uruguay_conf_chile.doc

Moreno, Luis (2007). Lucha contra la pobreza en América Latina: ¿selectividad y universalismo?, Consejo Superior de Investigaciones Científicas (CSIC), Unidad de Políticas Comparadas, España. Disponible desde Internet: http://www.iesam. csic.es/doctrab2/dt-0705.pdf

Oficina del Alto Comisionado de las Naciones Unidas para los Derechos Humanos, OACNUDH (2004). Los Derechos Humanos y la reducción de la pobreza: un marco conceptual. Naciones Unidas, Nueva York y Ginebra.

Oficina del Alto Comisionado de las Naciones Unidas para los Derechos Humanos, OACNUDH (2006). Principios y directrices para la integración de los Derechos Humanos en las estrategias de reducción de la pobreza. Disponible desde Internet: http:// www.reliefweb.int/rw/lib.nsf/db900SID/PANA7DGFWN?OpenDocument

Ovando, Vladimir "Otra mirada desde el Puente" (2007). Disponible desde Internet: http://www.politicapublica. cl/2007/10/10/otra-mirada-desde-el-puenteparte-33-por-vladimir-ovando/ 
Ruiz Jiménez, Laura (1008). “Cohesión social y lucha contra la pobreza: un balance de la políticas sociales en América Latina". Instituto Universitario de Investigación Ortega y Gasset. Disponible desde Internet: http://www.ceipaz.org/images/ contenido/06.\%20laura\%20ruiz\%20jimenez.pdf

Ruiz -Tagle, Pablo (2005). Pobreza y creación de derechos fundamentales. Sistema económico latinoamericano y del Caribe, SELA, Seminario Derecho y Pobreza, Panel 2: Las estrategias institucionales para erradicar la pobreza, Río de Janeiro, Brasil.

Sojo, Ana (2006). Vulnerabilidad social aseguramiento y diversificación de riesgos en América Latina y el Caribe, Revista de la CEPAL Nº 80.

MIDEPLAN, 2007. Tomado de: http://www.mideplan.cl/final/categoria. php?secid=1Ecatid=112 (Consultado el 3/10/2008). 